\title{
A Domain Knowledge Based Approach for Medical Image Retrieval
}

\author{
Haiwei Pan \\ College of Computer Science and Technology \\ Harbin Engineering University Harbin, China \\ heaven_007cn@yahoo.com.cn \\ Xiaolei Tan Qilong Han Guisheng Yin \\ College of Computer Science and Technology \\ Harbin Engineering University Harbin, China \\ \{tanxiaolei, hanqilong, yinguisheng\}@hrbeu.edu.cn
}

\begin{abstract}
The high incidence of brain disease, especially brain tumor, has increased significantly in recent years. It is becoming more and more concernful to discover knowledge through mining medical brain image to aid doctors' diagnosis. Image mining is the important branch of data mining. It is more than just an extension of data mining to image domain but an interdisciplinary endeavor. Image clustering and similarity retrieval are two basilic parts of image mining. In this paper, we introduce a notion of image sequence similarity patterns (ISSP) for medical image database. ISSP refer to the longest similar and continuous sub-patterns hidden in two objects each of which contains an image sequence. These patterns are significant in medical images because the similarity for two medical images is not important, but rather, it is the similarity of objects each of which has an image sequence that is meaningful. We design the new algorithms with the guidance of the domain knowledge to discover the possible Space-Occupying Lesion (PSO) in brain images and ISSP for similarity retrieval. Our experiments demonstrate that the results of similarity retrieval are meaningful and interesting to medical doctors.
\end{abstract}

Index Terms-Data mining; image mining; similarity retrieval; domain knowledge

\section{INTRODUCTION}

Advances in image acquisition and storage technology have led to tremendous growth in very large and detailed image databases [1]. A vast amount of image data is generated in our daily life and each field, such as medical image (CT images, ECT images and MR images etc), satellite images and all kinds of digital photographs. These images involve a great number of useful and implicit information that is difficult for users to discover.

Image mining can automatically discover these implicit information and patterns from the high volume of images and is rapidly gaining attention in the field of data mining. Image mining is more than just an extension of data mining to image domain. It is an interdisciplinary endeavor that draws upon computer vision, image processing, image retrieval, machine learning, artificial intelligence, database and data mining, etc. While some of individual fields in themselves may be quite matured, image mining, to date, is just a growing research focus and is still at an experimental stage. Research in image mining can be broadly classified to two main directions: (1) domain-specific applications; (2) general applications [2]. The focus in the first direction is to extract the most relevant image features into a form suitable for data mining $[4,8,9]$ and the latter is to generate image patterns that may be helpful in understanding of the interaction between high level human perceptions of image and low level image features $[1,10]$. Data mining in medical images belongs to the first direction.

Brain tissue is human's advanced nerve center, so its function is particularly important. The disease affecting the brain has received much attention in the domain of medicine. In China, about 40,000 to 60,000 persons suffer from brain tumor every year and about $16 \%$ of these persons are children. Especially during these years, the incidence of brain disease (especially brain tumor) has increased significantly and the quality of human's living even their lives has been endangered greatly. Therefore, the early diagnosis of brain diseases is becoming more and more crucial and is directly working on patients' treatment. That is why data mining in medical images for assisting medical staff is so significant. Furthermore, it is a greater challenge because of referring to the special domain.

Computerized Tomography (CT) is one of the most important techniques that are used to diagnose by medical doctors. Brain CT scan of each patient (as an object below) is an image sequence in which each one is an image of a layer every a few millimeters from calvaria. There exists a certain spatial relationship between images in the sequence. We will try to discover knowledge from this kind of image dataset by means of data mining technique.

This paper presents a new method to retrieve similar objects each of which includes an image sequence. The novelty includes three directions. The first is to make use of medical domain knowledge efficiently to guide data mining. The second is that we utilize two different clustering algorithms on pixels to discover the possible brain diseases that are called Space-Occupying Lesion 
(SO) by doctors, as shown in figure 1(c). The third is that we introduce a notion of image sequence similarity patterns ISSP for similarity retrieval.

The rest of the paper is organized as follows: section 2 is the statement of problem. Pre-processing is presented in section 3 and Detecting PSO Based on Pixel's Clustering is introduced in section 4. Section 5 presents Similarity Retrieval Based On ISSP. Conclusions and future research are presented in section 6.

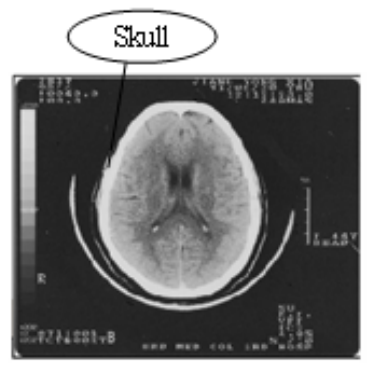

(a) Normal object's brain image before preprocessing

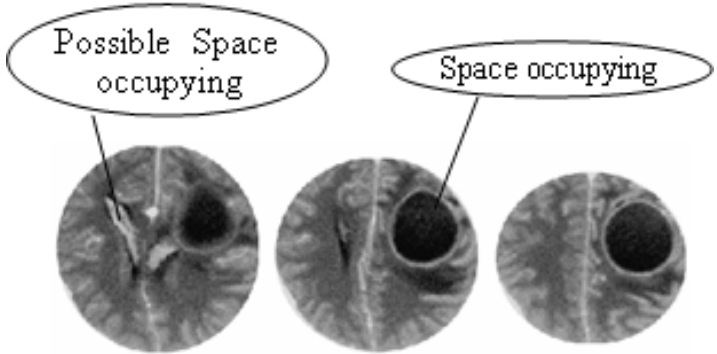

(c) An abnormal object's brain image sequence after preprocessing, further from calvaria from the first to the last

Figure 1: An example of normal and abnormal brain image

\section{STATEMENT OF PROBLEM}

At present, the main work of data mining on medical images [3,4,5,6,7,12,13] has two characteristics: (1) research content is the images in the medical image database, not the objects with medical images. For example, it is possible to classify images in the same object into the different class because they always have different morphological SO. This determines the type of knowledge that will be mined; (2) research method is to extract features from images to form feature attributes and use data mining on these attributes, not to consider the fundamental element - pixel's significance. In fact, medical doctors make a diagnosis mainly according to medical knowledge and the tone of pixels. Figure 2 shows the framework of these precious works, where $\mathrm{IM}_{\mathrm{i}}$ is an image and $F_{i j}$ is a feature in $\mathrm{IM}_{\mathrm{i}}$. Also, these work paid little attention to the guidance effect of domain knowledge to data mining.

Our research content is objects each of which contains a series of images and the images from different objects maybe have different intensity, see figure 3. The images

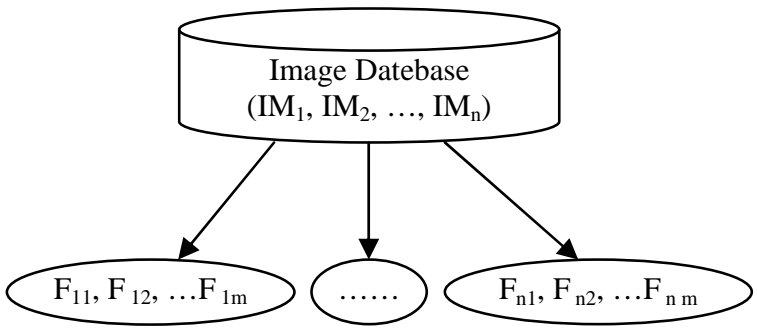

Figure 2: Framework of Precious Works. $\mathrm{IM}_{\mathrm{n}}$ is an image and $F_{n m}$ is one of the features extracted from $\mathrm{IM}_{n}$.

from one object have the same structure but come from the different layers of a brain. They have similar pixel density distribution and it is possible for two spatialadjacent images to be similar in one object, see figure 1(c). Each image contains some PSOs that also have a spatial relationship in an image. Therefore, the objects with the similar image sequence patterns (ISP) should have similar clinical manifestations. It is very helpful to assist medical doctors to make a diagnosis.

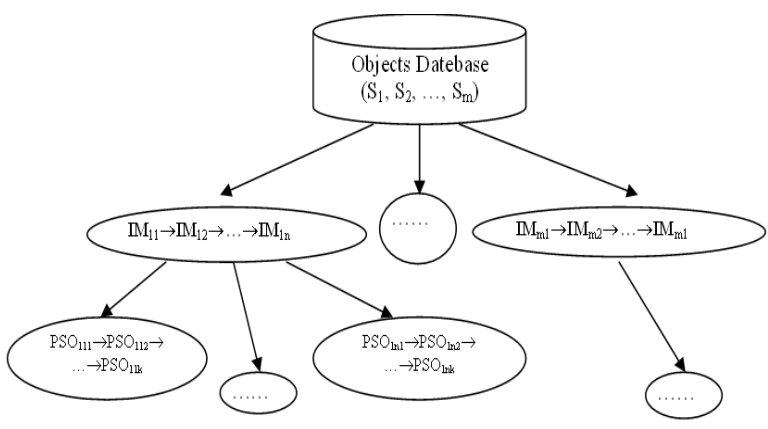

Figure 3: Framework of Our Work

\section{PRE-PROCESSING}

Since the images we got were raw CT scans that were scanned at different illumination conditions, some of them appeared too bright and some were too dark. We should digitize them to no loss, no compression and 256 gray scale images through special medical scanner. As brain CT scan of each object comprises several or more than ten images and there is a certain spatial relationship between these images, this greatly increases the quantity and complexity of data that is to be processed.

In preprocessing, our work uses domain knowledge effectively to remove the noisy data. A brain CT image mainly consists of three parts: noisy data, skull and cerebrum. The noisy data includes the black background and some additive information on it, such as CT identification, date and patient's name etc. This information is not only helpless but revealing patient's privacy. We are only interested in cerebrum. So we use domain knowledge (for short DK1) and cropping technique of image processing to gain it.

DK1 --- human's brain skull has the highest density and surrounds the cerebrum. 
Table 1: Each object is formed as a record in the table after its images are preprocessed.

\begin{tabular}{|c|c|}
\hline ID & IM \\
\hline 001 & $001.01 \rightarrow 001.02 \rightarrow 001.03 \rightarrow \ldots \rightarrow 001.07$ \\
\hline 002 & $002.01 \rightarrow 002.02 \rightarrow 002.03 \rightarrow \ldots \rightarrow 002.13$ \\
\hline$\ldots$ & $\ldots \ldots$. \\
\hline $\mathrm{n}$ & n. $01 \rightarrow \mathrm{n} .02 \rightarrow \mathrm{n} .03 \rightarrow \ldots \rightarrow \mathrm{n} .10$ \\
\hline
\end{tabular}

That is, the skull is a cricoid area in the image with the whitest pixels that separate cerebrum from the noisy data, see figure 1(a). It becomes easy to remove the noisy data by using cropping technique and keep the interesting region with the guidance of DK1. After image preprocessing, all the objects are formed as follows, see table 1. Each object has a unique identification (ID) and its image part (IM) is a preprocessed image sequence where every code is composed of id, a dot and the image sequence number, and the arrow represents the spatial relationship.

\section{DETECTING POSSIBLE SO (PSO) BASED ON PIXEL'S CLUSTERING}

The following domain knowledge (for short DK2) is used to direct clustering algorithm.

(1) The normal persons have nearly the same brain structure that is evident to be lateral symmetry. That is, the distribution of density in the left hemisphere of the brain is almost identical with the right, see figure 1 (b). If there is SO in either side, its density will change and destroy the symmetry;

(2) If one object has SO, it is more possible that this SO will be shown in some continuous images, see figure 1 (c).

\subsection{Basic Definitions}

Let $\mathrm{S}=\left\{\mathrm{S}_{\mathrm{i}} \mid \mathrm{i}=1 \ldots \mathrm{m}\right\}$ be object set; Let $\mathrm{S}_{\mathrm{i}}=\left\{\mathrm{IM}_{\mathrm{i} 1}, \mathrm{IM}_{\mathrm{i} 2}, \ldots\right.$, $\left.\mathrm{IM}_{\mathrm{in}}\right\}$ be object or ordered image set, where

(1) $\mathrm{IM}_{\mathrm{i} 1}$ and $\mathrm{IM}_{\mathrm{in}}$ are the nearest and farthest image from calvaria;

(2) For any $\mathrm{IM}_{\mathrm{i} 1}, \mathrm{IM}_{\mathrm{i} 2}, \ldots, \mathrm{IM}_{\mathrm{ip}}, \mathrm{IM}_{\mathrm{ip}}$ must be the farthest image from calvaria;

For any preprocessed image $\mathrm{IM}_{\mathrm{p}}$, it is halved by brain midline (shown as figure 1(b)) and is composed of two parts: $\operatorname{IM}_{\mathrm{p}}(\mathrm{L})$ and $\mathrm{IM}_{\mathrm{p}}(\mathrm{R})$ present the left and right hemisphere image respectively.

For any $\mathrm{IM}_{\mathrm{p}}$ and $\mathrm{IM}_{\mathrm{j}}$, they are adjacent if

(1) $\mathrm{IM}_{\mathrm{p}} \in \mathrm{S}_{\mathrm{i}}$ and $\mathrm{IM}_{\mathrm{j}} \in \mathrm{S}_{\mathrm{i}}$;

(2) $p=j+1$ or $p=j-1$;

Definition 1. Pixel set of $I_{p}$ is defined as $P=\left\{p_{i} \mid p_{i}\right.$ is the pixel with coordinate $\left(\mathrm{x}_{\mathrm{i}}, \mathrm{y}_{\mathrm{i}}\right)$ in the image $\left.\mathrm{IM}_{\mathrm{p}}\right\}, \mathrm{P}(\mathrm{L})$ and $P(R)$ are pixel set of $\mathrm{IM}_{\mathrm{p}}(\mathrm{L})$ and $\mathrm{IM}_{\mathrm{p}}(\mathrm{R})$ respectively.

According to the symmetry of the brain structure in DK2, we assume that the number of pixels in $\mathrm{IM}_{\mathrm{p}}(\mathrm{L})$ and $\mathrm{IM}_{\mathrm{p}}(\mathrm{R})$ are equal. That is, $|\mathrm{P}(\mathrm{L})|=|\mathrm{P}(\mathrm{R})|=|\mathrm{P}| / 2$. For any $\mathrm{p}_{\mathrm{li}} \in \mathrm{P}(\mathrm{L}), \mathrm{p}_{\mathrm{rj}} \in \mathrm{P}(\mathrm{R})$, they are symmetric pixel if the line between $\mathrm{p}_{\mathrm{li}}$ and $\mathrm{p}_{\mathrm{rj}}$ is halved vertically by brain midline. They are denoted as $\mathrm{p}_{\mathrm{li}}$ and $\mathrm{p}_{\mathrm{ri}}$ below.

Definition 2. We partition all pixels in pixel set $P$ into $\mathrm{m}$ blocks. Pixels in the same block have the same grey level and pixels in the different blocks have the different grey level. Let $G(P)=\left\{g_{1}, g_{2}, \ldots, g_{m}\right\}$ be P's grey-scale (GS) set if $G(P)$ is an ascending sort set of $g_{1}$ ', $\mathrm{g}_{2}$ ', ..., $\mathrm{g}_{\mathrm{m}}$ ' and $\mathrm{g}_{\mathrm{i}}$ ' is grey level of pixels in the $\mathrm{i}^{\text {th }}$ block, where $g_{i}(i=1, \ldots, m)$ is the $i^{\text {th }} G S, g_{1}$ ' and $g_{m}$ ' are P's minimum and maximum GS respectively. The GS of pixel $p_{i}$ is denoted as $g\left(p_{i}\right)$. we call $g_{\text {mean }}(P)$ the mean GS if

$$
\mathrm{g}_{\text {mean }}(\mathrm{P})=\sum_{\mathrm{i}=1}^{|\mathrm{P}|} \mathrm{g}\left(\mathrm{p}_{\mathrm{i}}\right) /|\mathrm{P}|
$$

Definition 3. For any $\mathrm{P}$ and distance function DisA $=\left|g_{k}-g_{\text {mean }}(P)\right|$, mid-value GS is a middle value in the GS set that minimizes DisA. Mid-value GS set is a set of mid-value GS.

Theorem 1 Mid-value GS set includes not more than two values.

Proof The value of DisA has two possibilities:

(1) If $g_{k}-g_{\text {mean }}(P)<0$, then DisA $=g_{\text {mean }}(P)-g_{k}$;

(2) If $g_{k}-g_{\text {mean }}(P) \geq 0$, then DisA $=g_{k}-g_{\text {mean }}(P)$;

In the first case, if there exist more than two GS that make the value of DisA be a certain minimum $\Upsilon$ : $g_{1}$, $\mathrm{g}_{2}{ }^{\prime}, \ldots, \mathrm{g}_{\mathrm{k}}{ }^{\prime}(\mathrm{k}>2)$, then they must satisfy the equation $\mathrm{g}_{1}{ }^{\prime}=\mathrm{g}_{2}{ }^{\prime}=\ldots=\mathrm{g}_{\mathrm{k}}$ '. This doesn't agree with the definition of GS set. Therefore, it is only one GS that minimizes DisA in the first case, denoted as g mida. $_{\text {. }}$

Case 2 is proved similarly and thus it is also only one GS that minimizes DisA, denoted as $\mathrm{g}_{\text {midb. }}$.

If $\mathrm{g}_{\text {mean }}(\mathrm{P})-\mathrm{g}_{\text {mida }}=\mathrm{g}_{\text {midb }}-\mathrm{g}_{\text {mean }}(\mathrm{P})$, then mid-value set includes two elements. Otherwise, it only includes one of $g_{\text {mida }}$ and $g_{\text {midb }}$ that minimizes DisA.

For any $\mathrm{P}$, if

(1) Mid-value GS set includes one element $g_{\text {mid }}$, and $\mathrm{g}_{\mathrm{s}}$ is the minimum value between $\mathrm{g}_{\text {mean }}$ and $\mathrm{g}_{\text {mid; }}$;

(2) Mid GS set includes two elements, $g_{s}$ is the minimum value between these two values;

$\mathrm{g}_{s}$ is called Benchmark GS and another one is denoted as $g_{s}$ '.

Definition 4. For pixel set $P$, let

$\mathrm{g}^{(\mathrm{l})}=\left\{\mathrm{g}_{\mathrm{i}}\left|\mathrm{g}_{1} \leq \mathrm{g}_{\mathrm{i}} \leq \mathrm{g}_{1}+\right| \mathrm{g}_{1}-\mathrm{g}_{\mathrm{s}} \mid / 2\right\}$ be low bound GS;

$\mathrm{g}^{(\mathrm{h})}=\left\{\mathrm{g}_{\mathrm{i}}\left|\mathrm{g}_{\mathrm{m}}-\right| \mathrm{g}_{\mathrm{m}}-\mathrm{g}_{\mathrm{s}}{ }^{\prime} \mid / 2 \leq \mathrm{g}_{\mathrm{i}} \leq \mathrm{g}_{\mathrm{m}}\right\}$ be high bound GS;

$\mathrm{g}^{(\mathrm{b})}=\mathrm{g}^{(\mathrm{l})} \cup \mathrm{g}^{(\mathrm{h})}$ be bound GS;

For pixel set $P$, let

$\mathrm{P}^{(1)}=\left\{\mathrm{p}_{\mathrm{i}} \mid \mathrm{g}\left(\mathrm{p}_{\mathrm{i}}\right) \in \mathrm{g}^{(\mathrm{l})}\right\}$ be low bound pixel set;

$\mathrm{P}^{(\mathrm{h})}=\left\{\mathrm{p}_{\mathrm{i}} \mid \mathrm{g}\left(\mathrm{p}_{\mathrm{i}}\right) \in \mathrm{g}^{(\mathrm{h})}\right\}$ be high bound pixel set;

$\mathrm{P}^{(\mathrm{b})}=\mathrm{P}^{(\mathrm{l})} \cup \mathrm{P}^{(\mathrm{h})}$ be bound pixel set and pixels in $\mathrm{P}^{(\mathrm{b})}$ are bound pixels.

Definition 5. $\Delta \mathrm{g}(\mathrm{P})$ is $\mathrm{IM}_{\mathrm{p}}$ 's difference set if for any symmetrical pixel $\mathrm{p}_{\mathrm{li}}$ and $\mathrm{p}_{\mathrm{ri}}$ in $\mathrm{P}, \Delta \mathrm{g}(\mathrm{P})=\left\{\Delta \mathrm{g}_{\mathrm{i}} \mid \Delta \mathrm{g}_{\mathrm{i}}=\mathrm{g}\left(\mathrm{p}_{\mathrm{li}}\right)-\right.$ $\left.\mathrm{g}\left(\mathrm{p}_{\mathrm{ri}}\right), \mathrm{i}=1,2, \ldots,|\mathrm{p}| / 2\right\}$.

Definition 6. For any image sequence $<\mathrm{IM}_{\mathrm{ij}}, \ldots$, $\mathrm{IM}_{\mathrm{ik}}>$, if

(1) only the first and last image $\mathrm{IM}_{\mathrm{ij}}$ and $\mathrm{IM}_{\mathrm{ik}}$ have one adjacent IM;

(2) other $\mathrm{IM}_{\mathrm{ip}}$ (if existed) has two adjacent IM;

we called that it has the property of continuity. Image sequence $<\mathrm{IM}_{\mathrm{ij}}, \ldots, \quad \mathrm{IM}_{\mathrm{ik}}>$ has the property of discontinuity if it can't satisfy the property of continuity.

Definition 7. For any pixel $\mathrm{p}_{\mathrm{i}}$ and a certain integer $\varepsilon$, the assemble of pixels is called $\varepsilon$-adjacent area $(\varepsilon-\mathrm{AA})$ if distance between $\mathrm{p}_{\mathrm{i}}$ and pixels in the assemble is not more than $\varepsilon . \mathrm{P}_{\mathrm{i}}$ is core pixel (c-pixel) if its $\varepsilon$-AA involves no less than MP pixels that satisfy some 
conditions. $\mathrm{P}_{\mathrm{i}}$ is immediate density reachable from $\mathrm{p}_{\mathrm{j}}$ if $\mathrm{p}_{\mathrm{i}}$ is in the $\varepsilon$-AA of $\mathrm{p}_{\mathrm{j}}$ and $\mathrm{p}_{\mathrm{j}}$ is c-pixel. $\mathrm{P}_{1}$ and $\mathrm{p}_{\mathrm{k}}$ are density reachable if for some pixels $\mathrm{p}_{1}, \mathrm{p}_{2}, \ldots, \mathrm{p}_{\mathrm{k}}$, any pixel $\mathrm{p}_{\mathrm{i}+1}$ is immediate density reachable from $\mathrm{p}_{\mathrm{i}} . \mathrm{P}_{\mathrm{i}}$ and $\mathrm{p}_{\mathrm{j}}$ are density connective if there exists pixel $\mathrm{p}_{\mathrm{k}}$ that is density reachable from not only $\mathrm{p}_{\mathrm{i}}$ but also $\mathrm{p}_{\mathrm{j}}$.

\subsection{Clustering Algorithm with the Guidance of DK2}

It is very crucial step for medical doctors to determine whether there is a Space-Occupying Lesion or not in the brain images. In this paper, we use clustering method on the pixels of images to detect the PSO. Firstly, for any $\mathrm{IM}_{\mathrm{p}}$, we compute to get the $\mathrm{IM}_{\mathrm{p}}$ 's difference set $\Delta \mathrm{g}(\mathrm{P})$, then sort the absolute value of each element in $\Delta \mathrm{g}(\mathrm{P})$ to yield the set $\Delta \mathrm{g}^{\prime}(\mathrm{P})=\left\{\left|\Delta \mathrm{g}_{\mathrm{i}}\right| \mid \Delta \mathrm{g}_{\mathrm{i}} \in \Delta \mathrm{g}(\mathrm{P})\right.$ and for any $\left|\Delta \mathrm{g}_{\mathrm{i}}\right|$, it must be maximal in the former i elements $\}$. Each element of $\Delta g^{\prime}(\mathrm{P})$ is regarded as an atomic cluster and hierarchical clustering from bottom to top will not stop in the light of the following similarity function of difference between pixels' GS until the number of clusters is equal to a specified value $\mathrm{k}$.

$\operatorname{similarity}\left(C_{i}, C_{j}\right)=\min \left|T_{i}-T_{j}\right|$, where $T_{i}$ and $T_{j}$ are mean value of all $\left|\Delta \mathrm{g}_{\mathrm{i}}\right|$ in cluster $\mathrm{C}_{\mathrm{i}}$ and $\mathrm{C}_{\mathrm{j}}$ respectively. The algorithm is as follows:

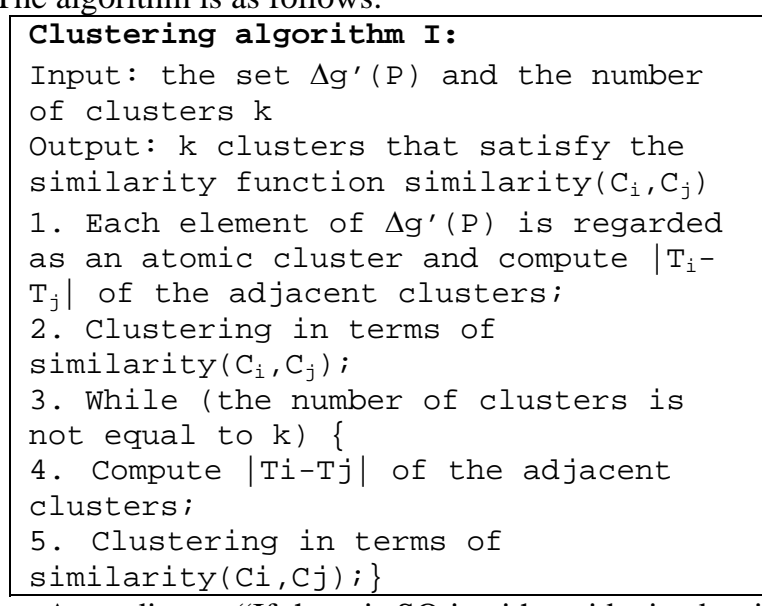

According to "If there is SO in either side, its density will change and destroy the symmetry" in DK2, we can deduce that if there exists SO in the image, then pixels' GS of SO should change and the value of the elements corresponding to these pixels in $\Delta g^{\prime}(P)$ will be much greater than zero. Otherwise, the value of these corresponding elements in $\Delta \mathrm{g}^{\prime}(\mathrm{P})$ will approximate zero. Therefore, we set the number of clusters to 2 as the termination condition. The first step of Clustering algorithm I scans $|\mathrm{P}| / 2$ elements in the set $\Delta \mathrm{g}^{\prime}(\mathrm{P})$ one time and time complexity is $\mathrm{O}(|\mathrm{P}| / 2)$. The second step is to select the minimum and time complexity is $\mathrm{O}(|\mathrm{P}| / 2)$, too. In the third step, the loop times is related to the speed of clustering. The worst case, only two clusters is clustered to a bigger cluster in each loop. The number of time is $|\mathrm{P}| / 2-3$ and time complexity of the $4^{\text {th }}$ and $5^{\text {th }}$ step is $\mathrm{O}(\mid \mathrm{P} / 2-\mathrm{i})$, where $\mathrm{i}$ is the $\mathrm{i}^{\text {th }}$ loop. Accordingly, the $3^{\text {rd }}$ and $5^{\text {th }}$ step for the worst case need $(n-3)(n+2) / 2$ operations and time complexity is $\mathrm{O}\left(\mathrm{n}^{2}\right)$.

According to the symmetry of the brain structure, we single out the cluster with the greater $\left|\Delta \mathrm{g}_{\mathrm{i}}\right|$ from clustering algorithm I (denoted as high difference cluster) to be the main study objects of the next step. It means that data size to be processed may be reduced. For Each $\Delta \mathrm{g}_{\mathrm{i}}$, there are two corresponding symmetric pixels $\mathrm{p}_{\mathrm{li}}$ and $\mathrm{p}_{\mathrm{ri}}$. All symmetric pixels $\mathrm{g}\left(\mathrm{p}_{\mathrm{li}}\right)$ and $\mathrm{g}\left(\mathrm{p}_{\mathrm{ri}}\right)$ in the high difference cluster are judged to be whether bound GS or not, then bound pixel set is generated.

Next, the based-on density clustering method is utilized to re-cluster these bound pixel set and determine the location and size of SO in each brain image. The algorithm is as follows:

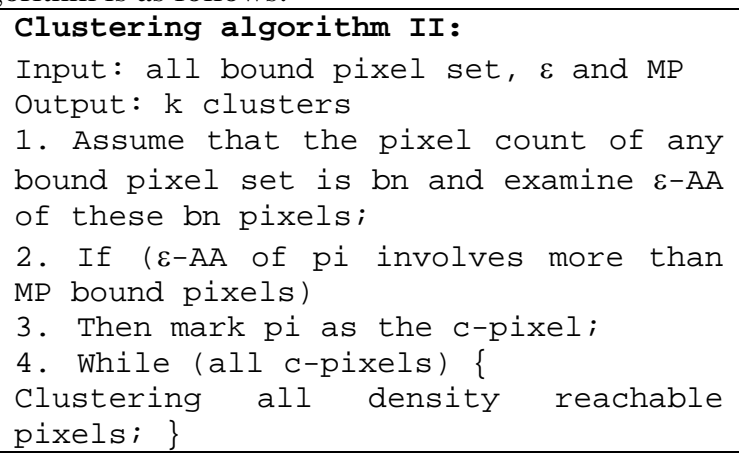

For $\varepsilon$ and MP in clustering algorithm II, we specify their value through learning on the normal object's brain images. The learning process is as follows: (1) run the first clustering on the normal object's $\mathrm{IM}_{\mathrm{p}}$ and achieve the bound pixel set; (2) count (not clustering) on the bound pixel sets which, in fact, are noisy data but not SO, and compute the maximum of the radius and the count of all bound pixel set which are the greatest lower bound of $\varepsilon$ and MP. Time complexity of this algorithm is $\mathrm{O}(\mathrm{nlogn})$. The k clusters generated from this algorithm are $\mathrm{k}$ PSOs.

\section{SIMILARITY RETRIEVAL BASED ON ISSP}

In this section, we will: (1) discover the image sequence pattern (ISP) of one object; (2) discover the image sequence similarity patterns (ISSP) of two objects.

\subsection{Mining ISP of one object}

The whole PSO in an image can be found using the above algorithm. For these PSOs, we denote each PSO as the following: $<\mathrm{H}(\mathrm{L}),\left(\mathrm{x}_{\mathrm{i}}, \mathrm{y}_{\mathrm{i}}\right),\left(\mathrm{x}_{\mathrm{a} 1}, \mathrm{x}_{\mathrm{a} 2}\right),\left(\mathrm{y}_{\mathrm{b} 1}, \mathrm{y}_{\mathrm{b} 2}\right)>$, where $\mathrm{H}(\mathrm{L})$ represents the high (low) bound $\mathrm{GS},\left(\mathrm{x}_{\mathrm{i}}, \mathrm{y}_{\mathrm{i}}\right)$ is the coordinate of the center of this PSO, $\left(x_{a 1}, x_{a 2}\right)$ and $\left(\mathrm{y}_{\mathrm{b} 1}, \mathrm{y}_{\mathrm{b} 2}\right)$ are the max and min $\mathrm{x}$ and $\mathrm{y}$ coordinate of the PSO. They are computed by the following formula:

$$
\begin{array}{lll}
\mathrm{x}_{\mathrm{i}}=\frac{1}{k} \sum_{j=1}^{k} x_{j} & \text { (1) } & \mathrm{y}_{\mathrm{i}}=\frac{1}{k} \sum_{j=1}^{k} y_{j} \\
\mathrm{x}_{\mathrm{a} 1}=\max _{j=1}^{k}\left(x_{j}\right) & \text { (3) } & \mathrm{x}_{\mathrm{a} 2}=\min _{j=1}^{k}\left(x_{i j}\right) \\
\mathrm{y}_{\mathrm{b} 1}=\max _{j=1}^{k}\left(y_{j}\right) & \text { (5) } & \mathrm{y}_{\mathrm{b} 2}=\min _{j=1}^{k}\left(y_{j}\right)
\end{array}
$$

We take the center of the brain as the origin of coordinates. If $x_{i} \leq 0$, then this PSO is in $\mathrm{IM}(\mathrm{L})$. Otherwise, it is in IM(R).

Definition 8. $\mathrm{PSO}_{\mathrm{k}}$ is prior to $\mathrm{PSO}_{\mathrm{j}}$ if for $\mathrm{PSO}_{\mathrm{k}}=$ $<\mathrm{H}(\mathrm{L}),\left(\mathrm{x}_{\mathrm{k}}, \mathrm{y}_{\mathrm{k}}\right),\left(\mathrm{x}_{\mathrm{ka} 1}, \mathrm{x}_{\mathrm{ka} 2}\right),\left(\mathrm{y}_{\mathrm{kb} 1}, \mathrm{y}_{\mathrm{kb} 2}\right)>$ and $\mathrm{PSO}_{\mathrm{j}}=$ 
$<\mathrm{H}(\mathrm{L}),\left(\mathrm{x}_{\mathrm{j}}, \mathrm{y}_{\mathrm{j}}\right),\left(\mathrm{x}_{\mathrm{ja} 1}, \mathrm{x}_{\mathrm{ja} 2}\right),\left(\mathrm{y}_{\mathrm{jb} 1}, \mathrm{y}_{\mathrm{jb} 2}\right)>$, they satisfy one of the three prior conditions:

(a) $\mathrm{x}_{\mathrm{k}}<\mathrm{x}_{\mathrm{j}}$; (b) $\mathrm{x}_{\mathrm{k}}=\mathrm{x}_{\mathrm{j}}$ and $\mathrm{y}_{\mathrm{k}}>\mathrm{y}_{\mathrm{j}}$; (c) $\mathrm{x}_{\mathrm{ka} 1} \leq \mathrm{x}_{\mathrm{ja} 1}$ and $\mathrm{x}_{\mathrm{ka} 2} \geq \mathrm{x}_{\mathrm{ja} 2}$; (d) $\mathrm{y}_{\mathrm{kb} 1} \leq \mathrm{y}_{\mathrm{jb} 1}$ and $\mathrm{y}_{\mathrm{kb} 2} \geq \mathrm{y}_{\mathrm{jb} 2}$;

We denote it as $\mathrm{PSO}_{\mathrm{k}}>>\mathrm{PSO}_{\mathrm{j}}$.

According to this priority, we describe PSO pattern (PSOP) of each image as the following form: $\operatorname{PSOP}\left(\mathrm{IM}_{\mathrm{i}}\right)=<\mathrm{L}_{\mathrm{i} 1}, \mathrm{~L}_{\mathrm{i} 2}, \ldots, \mathrm{L}_{\mathrm{im}} ; \mathrm{R}_{\mathrm{i} 1}, \mathrm{R}_{\mathrm{i} 2}, \ldots, \mathrm{R}_{\mathrm{in}}>$

Where $L_{i m}$ and $R_{\text {in }}$ represent a PSO in $\operatorname{IM}_{i}(L)$ and $\mathrm{IM}_{\mathrm{i}}(\mathrm{R})$ respectively.

Definition 9. PSOP of two images is complete similar if $\left|\operatorname{PSOP}\left(\mathrm{IM}_{\mathrm{i}}\right)\right|=\left|\operatorname{PSOP}\left(\mathrm{IM}_{\mathrm{j}}\right)\right|$, and the corresponding $L_{i k}$ and $L_{j k}$ (or $R_{i k}$ and $R_{j k}$ ) have the same bound GS, locate the same part of the image (IM(L) or IM(R)) and satisfy the same prior condition. That is, if $\mathrm{L}_{\mathrm{ik}}$ and $\mathrm{L}_{\mathrm{i}(\mathrm{k}+1)}$ satisfy the prior condition (a) or (b), then $\mathrm{L}_{\mathrm{jk}}$ and $\mathrm{L}_{\mathrm{j}(\mathrm{k}+1)}$ must satisfy (a) or (b).

Definition 10. PSOP of two images is incomplete similar if cut one or more discontinuous PSO from one or both of these two images, then $\left|\operatorname{PSOP}\left(\mathrm{IM}_{\mathrm{i}}\right)\right|=$ $\left|\operatorname{PSOP}\left(\mathrm{IM}_{\mathrm{j}}\right)\right|$, and the corresponding $\mathrm{L}_{\mathrm{ik}}$ and $\mathrm{L}_{\mathrm{jk}}\left(\right.$ or $\mathrm{R}_{\mathrm{ik}}$ and $R_{j k}$ ) have the same bound GS, locate the same part of the image (IM(L) or IM(R)) and satisfy the same prior condition.

For each image of one object, it has a PSOP and maybe is different from the PSOP of the adjacent image. We compare the PSOP of all adjacent images and get a pattern sequence in which two adjacent patterns are not same. This pattern sequence is called image sequence pattern (ISP).

The algorithm of discovering ISP is as follows.

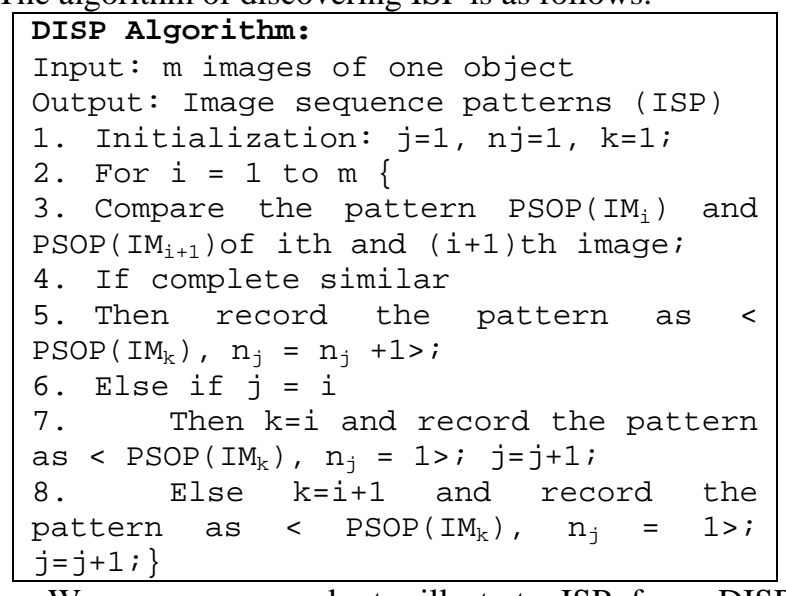

We use an example to illustrate ISP from DISP algorithm, see figure 4. Convenient for description, we denote ISP as the following form:

$$
\begin{aligned}
& \operatorname{ISP}\left(\mathrm{S}_{\mathrm{i}}\right)=\left\{<\mathrm{M}_{\mathrm{i}}\left(\mathrm{IM}_{\mathrm{i} 1}\right), \mathrm{n}_{1}>,<\mathrm{M}_{\mathrm{i}}\left(\mathrm{IM}_{\mathrm{i} 2}\right), \mathrm{n}_{2}>, \ldots,\right. \\
& \left.<\mathrm{M}_{\mathrm{i}}\left(\mathrm{IM}_{\mathrm{ik}}\right), \mathrm{n}_{\mathrm{k}}>\right\} \text {, or } \\
& \left\{<\mathrm{M}_{\mathrm{i}}, \mathrm{n}_{1}>,<\mathrm{M}_{\mathrm{i}}, \mathrm{n}_{2}>, \ldots,<\mathrm{M}_{\mathrm{i}}, \mathrm{n}_{\mathrm{k}}>\right\}
\end{aligned}
$$

Where for $\mathrm{j}=1 \ldots \mathrm{k}, \mathrm{M}_{\mathrm{j}}$ and $\mathrm{M}_{\mathrm{j}+1}$ are the distinct PSOP. $I_{i j}$ is the first image with the PSOP $M_{j}$ and $n_{j}$ is the number of the adjacent images with the same PSOP $\mathrm{M}_{\mathrm{j}}$.

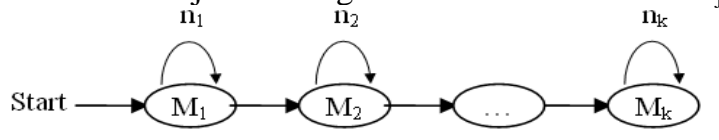

Figure 4: Patterns from Discovering ISP Algorithm
For ISP $\left(\mathrm{S}_{\mathrm{i}}\right)$ and $\operatorname{ISP}\left(\mathrm{S}_{\mathrm{j}}\right)$, ISSP of two objects refers to the longest similar and continuous sub-patterns that belongs to the ISP of each object. For example, ISP $\left(\mathrm{S}_{\mathrm{i}}\right)=\left\{<\mathrm{M}_{1}\right.$, $1>,\left\langle\mathrm{M}_{2}, 2>,\left\langle\mathrm{M}_{3}, 2>,\left\langle\mathrm{M}_{4}, 1>,\left\langle\mathrm{M}_{5}, 1>\right\}\right.\right.\right.$ and $\operatorname{ISP}\left(\mathrm{S}_{\mathrm{j}}\right)=\left\{<\mathrm{M}_{1}, 1>,<\mathrm{M}_{2}, 3>,<\mathrm{M}_{3}, 3>,<\mathrm{M}_{4}, 2>,<\right.$ $\left.M_{5}{ }^{\prime}, 1>,<M_{6}{ }^{\prime}, 1>\right\}$, if $M_{1}$ is similar to $M_{1}{ }^{\prime}, M_{3}$ similar to $M_{3}, M_{4}$ similar to $M_{4}$ ' and $M_{5}$ similar to $M_{6}$, then ISSP of these two objects is $\left\langle\mathrm{M}_{1}{ }^{\prime}, \mathrm{M}_{3}{ }^{\prime}, \mathrm{M}_{4}{ }^{\prime}, \mathrm{M}_{6}{ }^{\prime}>\right.$. Here, $\mathrm{M}_{3}{ }^{\text {' }}$ and $\mathrm{M}_{4}{ }^{\prime}$ are called continuous sup-pattern, and $\mathrm{M}_{1}{ }^{\prime}$ and $\mathrm{M}_{3}$ ' are discontinuous sup-pattern.

Since there are spatial relationship between all images in $S_{i}$, that is, for any $\mathrm{IM}_{\mathrm{i} 1}, \mathrm{IM}_{\mathrm{i} 2}, \ldots, \mathrm{IM}_{\mathrm{in}}, \mathrm{IM}_{\mathrm{i}(\mathrm{j}+1)}$ must be farther from calvaria than $\mathrm{IM}_{\mathrm{ij}}$, it is not necessary to retrieve the whole PSOPs of one object to find the similar pattern of a given $\mathrm{M}_{\mathrm{i}}$. For example, $\mathrm{M}_{1}$ is PSOP of the farthest image from calvaria of one object and $M_{p}$ is PSOP of the nearest image of another different object, it is not meaningful to compare $M_{1}$ and $M_{p}$ to find whether they are similar or not because $M_{1}$ and $M_{p}$ show the different parts of the brain. According to this, two rules are introduced to reduce the retrieval space and enhance the efficiency. For two objects $S_{i}$ and $S_{j}$, assumed that $\operatorname{ISP}\left(\mathrm{S}_{\mathrm{i}}\right)=\mathrm{m}$ and $\operatorname{ISP}\left(\mathrm{S}_{\mathrm{j}}\right)=\mathrm{n}$,

(1) if $m=n$, that is, the number of the PSOPs in these two objects is equal, then we only need to retrieve $\mathrm{M}_{\mathrm{i}-1}{ }^{\prime}, \mathrm{M}_{\mathrm{i}}$ ' and $\mathrm{M}_{\mathrm{i}+1}$ ' in $\operatorname{ISP}\left(\mathrm{S}_{\mathrm{i}}\right)$ to discover the similar patterns of $\mathrm{M}_{\mathrm{i}}$ in $\operatorname{ISP}\left(\mathrm{S}_{\mathrm{i}}\right)$.

(2) If $m<n$, then we only need to retrieve $M_{i}, M_{i+1}, \ldots$, $\mathrm{M}_{\mathrm{i}+\mathrm{n}-\mathrm{m}}$ 'in $\operatorname{ISP}\left(\mathrm{S}_{\mathrm{i}}\right)$ to discover the similar patterns of $\mathrm{M}_{\mathrm{i}}$ in $\operatorname{ISP}\left(\mathrm{S}_{\mathrm{i}}\right)$.

The algorithm of discovering ISSP is as follows.

\section{DISSP Algorithm:}

Input: ISP of two objects

Output: Image sequence

patterns (ISSP)

1. Initialization: $\mathrm{C}=\mathrm{NULL}, \mathrm{NC}=\mathrm{NULL}$;

2. Assumed that $\operatorname{ISP}\left(S_{i}\right)=m$ and $\operatorname{ISP}\left(S_{j}\right)=n$,

if $m \leq n$, start the following steps:

3. For $i=1$ to $m\{$

4. if $m=n$, then goto step (5); if $m<n$, then goto $\operatorname{step}(7)$;

5. Compare $M_{i}$ in $\operatorname{ISP}\left(S_{i}\right)$ with $M_{i-1}{ }^{\prime}, M_{i}{ }^{\prime}$ and $M_{i+1}^{\prime}$ in $\operatorname{ISP}\left(S_{j}\right)$. If discover a complete similar PSOP, $C=C \cup\left(M_{i} \rightarrow M_{j}\right)$. If there is no PSOP to compare in $\operatorname{ISP}\left(S_{j}\right)$, return step (3) to start the next iteration. If there is no complete similar PSOP to be discovered, goto step (6);

6. Compare $M_{i}$ in $\operatorname{ISP}\left(S_{i}\right)$ with $M_{i-1}{ }^{\prime}, M_{i}{ }^{\prime}$ and $M_{i+1}$ in $\operatorname{ISP}\left(S_{j}\right)$. If discover a incomplete similar PSOP, NC=NC $\cup\left(M_{i} \rightarrow M_{j}\right)$. If there is no PSOP to compare in $\operatorname{ISP}\left(S_{j}\right)$, return step (3) to start the next iteration;

7. Compare $M_{i}$ in $\operatorname{ISP}\left(S_{i}\right)$ with $M_{i}{ }^{\prime}$, $M_{i+1}{ }^{\prime}, \ldots, M_{i+n-m}$ in ISP $\left(S_{j}\right)$. If discover a complete similar PSOP, $C=C \cup\left(M_{i} \rightarrow M_{j}\right)$. If there is no PSOP to compare in $\operatorname{ISP}\left(S_{j}\right)$, return step (3) to start the next iteration. If there is no complete similar PSOP to be discovered, goto step (8);

8. Compare $M_{i}$ in $\operatorname{ISP}\left(S_{i}\right)$ with $M_{i}{ }^{\prime}$, $M_{i+1}, \ldots, M_{i+n-m}{ }^{\prime}$ in $\operatorname{ISP}\left(S_{j}\right)$. If discover a

\subsection{Mining ISSP of two objects}




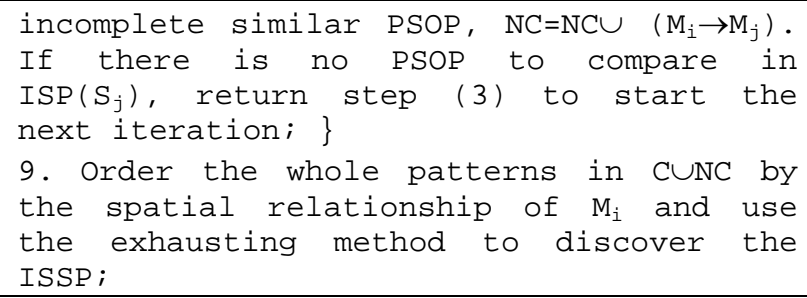

Since the above rules reduce the number of the PSOPs to be retrieved efficiently, the time cost of this algorithm mainly lies on the procedure of comparing the similarity of two PSOPs that depends on the count of the PSOs generated from the two algorithms in section 4.2. Therefore, the time complexity of this algorithm is $\mathrm{O}(\mathrm{km})$, where $\mathrm{k}$ is the number of the PSOs.

Definition 11. Two objects are similar if there exists ISSP in two objects.

We give an example to illustrate this algorithm. For two objects $S_{i}$ and $S_{j}, \operatorname{ISP}\left(S_{i}\right)=\left\{<M_{1}, 1>,<M_{2}, 2>,<M_{3}, 2>\right.$, $\left.<\mathrm{M}_{4}, 1>,<\mathrm{M}_{5}, 1>,<\mathrm{M}_{6}, 1>\right\}$ and $\operatorname{ISP}\left(\mathrm{S}_{\mathrm{j}}\right)=\left\{<\mathrm{M}_{1}, 1>\right.$, $<\mathrm{M}_{2}, 3>,<\mathrm{M}_{3}, 1>$, we use DISSP algorithm to get the results: $C=\left(M_{2}{ }^{\prime} \rightarrow M_{2}, M_{4}\right)$ and $\mathrm{NC}=\left(M_{1}{ }^{\prime} \rightarrow M_{1}, M_{3}\right) \cup$ $\left(\mathrm{M}_{3}{ }^{\prime} \rightarrow \mathrm{M}_{6}\right.$ ), where $\mathrm{C}$ and NC includes the complete and incomplete similar patterns respectively. Notice that $\left|\operatorname{ISP}\left(\mathrm{S}_{\mathrm{j}}\right)\right|<\left|\operatorname{ISP}\left(\mathrm{S}_{\mathrm{i}}\right)\right|$, we order $\mathrm{C} \cup N C$ by the spatial relationship of $\mathrm{M}_{\mathrm{j}}$ in $\operatorname{ISP}\left(\mathrm{S}_{\mathrm{j}}\right)$. The result is $\left(\mathrm{M}_{1}{ }^{\prime} \rightarrow \mathrm{M}_{1}, \mathrm{M}_{3}\right)$ $\cup\left(\mathrm{M}_{2}{ }^{\prime} \rightarrow \mathrm{M}_{2}, \mathrm{M}_{4}\right) \cup\left(\mathrm{M}_{3}{ }^{\prime} \rightarrow \mathrm{M}_{6}\right)$. By means of exhausting method, the final ISSP is $\left\langle\mathrm{M}_{1}, \mathrm{M}_{2}, \mathrm{M}_{6}>\right.$ and $<\mathrm{M}_{3}, \mathrm{M}_{4}, \mathrm{M}_{6}>$. Therefore, $\mathrm{S}_{\mathrm{i}}$ is similar to $\mathrm{S}_{\mathrm{j}}$.

\section{EXPERIMENTS}

To have access to real medical images is a very difficult undertaking due to legally privacy issues and management of hospital. But with some specialists' help and support, we got 103 pieces of precious data, which included 11 normal objects' CT scans and 92 abnormal objects data including CT scans and clinical data. Up to now, we have not found the method used on this kind of object dataset where each object has a brain image sequence. Therefore, we only give our experimental results.

\subsection{Preprocessing with the guidance of DK1}

For the preprocessing without the guidance of domain knowledge, the general method is cropping the images horizontally and vertically, see figure 5 (b). Figure 5 (c) is the preprocessed image with the guidance of DK1.

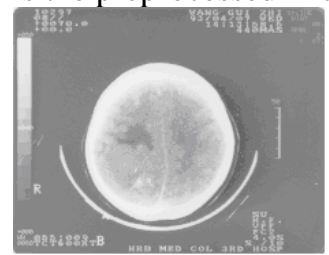

(a) Original Image

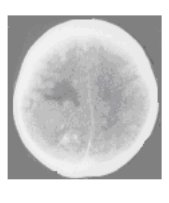

(b) Cropped Image Image with DK1

\subsection{Similarity Retrieval with the guidance of DK1}

We randomly sample 10 percent of normal objects and 10 percent of abnormal objects as the targets from the dataset and use our algorithm to retrieve the similar objects of each target from the whole dataset. The formulas for precision and recall are given below to evaluate our algorithm:

$$
\begin{aligned}
& P(p)=\frac{T P}{T P+F P} \\
& \mathrm{P}(\mathrm{n})=\frac{\mathrm{TN}}{\mathrm{TN}+\mathrm{FN}} \\
& \begin{array}{l}
\mathrm{R}(\mathrm{p})=\frac{\mathrm{TP}}{\mathrm{TP}+\mathrm{FN}} \\
\mathrm{R}(\mathrm{n})=\frac{\mathrm{TN}}{\mathrm{TN}+\mathrm{FP}}
\end{array}
\end{aligned}
$$

Where TP stands for true positives, FP for false positives, FN for false negatives, TN for true negatives, $\mathrm{P}(\mathrm{p})$ and $\mathrm{R}(\mathrm{p})$ for the precision and recall of similarity retrieval of abnormal objects, $\mathrm{P}(\mathrm{n})$ and $\mathrm{R}(\mathrm{n})$ for the precision and recall of similarity retrieval of normal objects. Figure 6 and figure 7 shows the experimental results of randomly sampling 5 times. We can observe that the precision and recall are very high for normal targets. Only one abnormal object is retrieved as the similar object of normal targets for each time because the tumor in the brain of this abnormal object is laterally symmetrical. While our clustering algorithms calculate the difference set, they ignore the tumor. One normal object is not retrieved for each time because his (her) brain images are too bright and some noisy dark pixels cause the false results.

The average precision of the abnormal targets is more than $60 \%$ but not very high. All retrieved objects are certainly the abnormal objects with tumor in their brain and the image sequence of them is similar to that of the target. The reason why not very high is that their concrete tumor kind is not the same as the target and the medical doctors made a different detailed diagnosis. But this demonstrates that our similarity retrieval algorithm based on ISSP can gain the similar image sequence of the target. The average recall, however, is very high. This illustrates that the results of our similarity retrieval method based on ISSP can include most of the similar objects with the targets. For example, there are 7 objects including the target itself that are actually similar to the target in the dataset and our method can find 10 similar objects in which 6 objects belong to the actually similar objects.

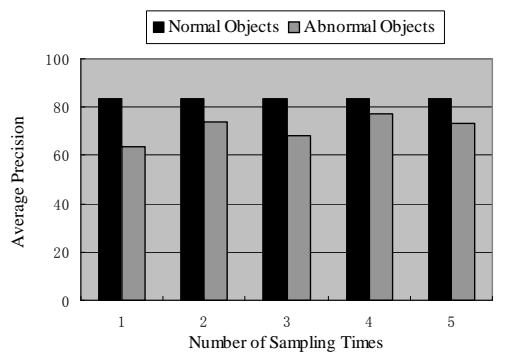

Figure 6: Average Precision of Similarity Retrieval when the Norman and Abnormal Objects as Targets

Figure 5: Digital brain image 


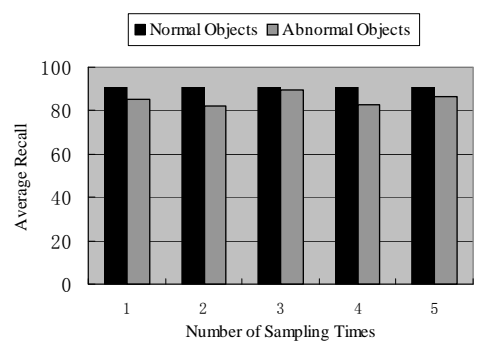

Figure 7: Average Recall of Similarity Retrieval when the Norman and Abnormal Objects as Targets

\section{CONCLUSIONS}

The high incidence of brain disease, especially brain tumor, has increased significantly in recent years. It is becoming more and more concernful to discover knowledge through mining medical brain image to aid doctors' diagnosis. In this paper, we firstly use two clustering algorithms to generate the possible SpaceOccupying Lesion, and then discover ISP for each object. Next, we introduce a notion of image sequence similarity patterns (ISSP) for medical image database. ISSP refer to the longest similar and continuous sub-patterns hidden in two objects each of which contains an image sequence. These patterns are significant in medical images because the similarity for most medical images is not important, but rather, it is the similarity of objects each of which has an image sequence that is meaningful. We design a new algorithm with the guidance of the domain knowledge to generate ISSP for similarity retrieval. Our experiments demonstrate that the guidance of domain knowledge is meaningful and the results of similarity retrieval are interesting to medical doctors.

Future research includes further contact with medical knowledge that will make us engage in studying the classification, clustering and retrieval methods in medical images. Also, we will combine the clinical data with images to study new methods to enhance the accuracy of similarity retrieval and classification.Hope you find the information in this template useful in the preparation of your submission.

\section{REFERENCES}

[1] Zaiane, O.R. et al. (1998). Mining MultiMedia Data. CASCON: Meeting of Minds.

[2] WYNNE HSU, MONG LI LEE, JI ZHANG. Image Mining: Trends and Developments. Journal of Intelligent Information Systems, 19:1, 7-23, 2002.

[3] Vasileios Megalooikonomou, Christos Davatzikos, Edward H. Herskovits. Mining Lesion-Deficit Associations in a Brain Image Database. KDD-99 San Diego CA USA.

[4] Wynne Hsu, Mong Li Lee, Kheng Guan Goh. Image Mining in IRIS: Integrated Retinal Information System. Proceedings of the ACM SIGMOD, May 2000, Dellas, Texas, U.S.A., pp. 593.

[5] Y. Liu, F. Dellaert, W.E. Rothfus, A. Moore, J. Schneider, and T. Kanade. Classification-Driven Pathological

Neuroimage Retrieval Using Statistical Asymmetry Measures. Proceedings of the Medical Imaging Computing and Computer Assisted Intervention Conference (MICCAI 2001), Utrecht, The Netherlands, October, 2001.

[6] Maria-Luiza Antonie, Osmar R. Zaiane, Alexandru Coman. Application of Data Mining Techniques for Medical Image Classification. Proceedings of the Second International Workshop on Multimedia Data Mining (MDM/KDD'2001).

[7] Osmar R. Zaiane, Maria-Luiza Antonie, Alexandru Coman. Mammography Classification by an Association Rule-based Classifier. Proceedings of the Third International Workshop on Multimedia Data Mining (MDM/KDD'2002).

[8] Fayyad, U.M., Djorgovski, S.G., and Weir, N. (1996). Automating the Analysis and Cataloging of Sky Surveys. Advances in Knowledge Discovery and Data Mining, 471493.

[9] Kitamoto, A. (2001). Data Mining for Typhoon Image Collection. In Second International Workshop on Multimedia Data Mining (MDM/KDD’2001).

[10] Ordonez, C. and Omiecinski, E. (1999). Discovering Association Rules Based on Image Content. In IEEE Advances in Digital Libraries Conference.

[11] Burl, MC et al. Mining For Image Content. In systems, Cybernetics, and Informatics / Information Systems: Analysis and Synthesis (1999).

[12] Soltanian-Zadeh H., Nezafat R., and Windham J.P.: "Is There Texture Information in Standard Brain MRI ?”, Proceedings of SPIE Medical Imaging 1999: Image Processing conference, San Diego, CA, Feb.1999.

[13] Barra V., Boire J-Y., "Tissue segmentation on MR Images of the Brain by Positivistic Clustering on a 3D Wavelet Representation.”, J. of Magnetic resonance Imaging, vol.11, pp. 267-278, 2000.

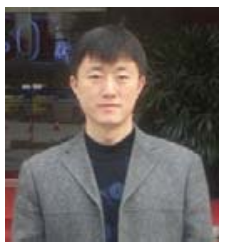

process.

I am currently an Assistant Professor in the College of Computer Science and Technology at Harbin Engineering University.

I teach "Algorithm Design and Analysis" and "Graph Theory" for undergraduate students, and "Combinatorial Mathematics" for graduate students. I have published more than 20 Conference papers and Journal papers. My research has been supported by the National Natural Science Foundation of China, the Natural Science Foundation of Heilongjiang Province,

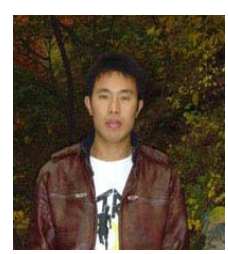
the Fundamental Research Funds for the Central Universities.

Xiaolei Tan, born in 1982, M.S.candidate. His current research interests focus on data mining.

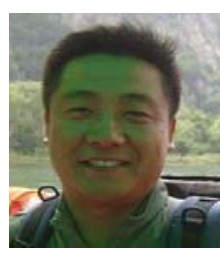

Qilong Han, born in 1974, Ph.d., associate professor. His current research interests include spatiotemporal database, protecting sensitive data, massive data process, real-time database. 